\title{
Meaningfulness in Physical Education: A Description of High School Students' Conceptions
}

\author{
Ang Chen \\ Arizona State University
}

\begin{abstract}
A theoretical framework distinguishing meaning and meaningfulness guided this study of high school students' conceptions of meaningfulness in physical education. A 9-dimensional meaningfulness construct was developed through analyzing former high school students' $(N=35)$ oral reflection on physical education. A 9-dimensional meaningfulness scale was prepared and administered to high school students $(N=698)$. The principal component analysis reduced the students' responses to a 6-dimensional construct: Social Bonding, Cultural Appreciation, Challenge, Tension Release, Fitness Development, and Self-Expression. The construct was modified through confirmatory factor analyses and had a Goodness of Fit Index of .91. The reconstruction demonstrated sophisticated internalization of perceived meaning by students. A MANOVA revealed that the students' conceptions of meaningfulness differentiated $(p<.05)$ based on gender, grade, and socioeconomic status. The findings suggest that a pluralistic perspective be considered in curriculum design, given the sophistication and differentiation of students' conceptions of meaningfulness in physical education.
\end{abstract}

The idea of helping students search for meaning is central to contemporary curriculum theories (Jewett, Bain, \& Ennis, 1995; Pinar, Reynolds, Slattery, \& Taubman, 1995). A primary assumption of these curriculum theories is that knowledge and skills are tentative and continually change with the changes in the world around us. Thus, the knowledge and skills are likely to be perceived as carrying different meanings for students. Based on this fundamental assumption, these curriculum theories further suggest that students are likely to learn knowledge and skills effectively if learning becomes personally meaningful through a process of exploring the relationship of its content with personal life (Cornbleth, 1990). The concepts of meaning and meaningfulness have been employed interchangeably in the literature in general curriculum studies (e.g., Cornbleth, 1990; Doll, 1992; MacDonald, 1988) and in physical education (e.g., Jewett, Bain, \& Ennis, 1995). This research, designed to investigate high school students' conceptions of meaningfulness in physical education, was guided by a theoretical framework that conceptually distinguishes these two important concepts.

Meaning and meaningfulness should be conceptualized as two related but independent concepts when the relevance of an activity to a person's life, no matter whether

Ang Chen is with the Department of Exercise Science and Physical Education at Arizona State University, P.O. Box 870701, Tempe, Arizona 85287-0701. 
it is learning, playing, or working, is central to a systematic inquiry. The concept of meaning encompasses and should be understood with three major components: purpose, intention arousal, and information (Csikszentmihalyi, 1990). Meaning is first defined by a person's conception of the purpose that an activity offers towards a perceived end. Based on the person's perception of its significance to his or her life, the purpose in the activity can be interpreted as either personally appropriate or inappropriate. Second, meaning is defined by an activity's power to arouse a person's intention to act when one encounters the activity, either actively engaging with or avoiding it. Third, meaning is defined by the information (knowledge or skill) that a person considers the activity to disseminate, either relevant or irrelevant. In other words, meaning is embedded also in identifying information on the basis of personal relevancy. Based on Csikszentmihalyi's interpretation, the construct of meaning of physical education should consist of (a) students' perceptions of purposes that the content requires them to achieve, (b) their intention to act when they encounter the content, and (c) the knowledge and skills in the content as perceived by the students.

The concept of meaningfulness, on the other hand, consists of three major components corresponding to the three elements defining the concept of meaning. First, it represents a person's desire to achieve the purpose. This desire demonstrates that the person has internalized the purpose of an activity into a goal significant to his or her own life. The personal desire indicates that, as Csikszentmihalyi (1990) pointed out, the purpose of content itself usually is not important, the process of internalizing or transforming it into a personal goal makes the purpose become meaningful to the person. Second, meaningfulness is characterized by an individual's striving to carry out the action that leads to achieving his or her goal. In this striving, "what counts is not so much whether a person actually achieves what she has set out to do; rather, it matters whether effort has been expended to reach the goal, instead of being diffused or wasted" (Csikszentmihalyi, 1990, p. 217). In other words, establishing a connection between action and goal is more important than simply engaging in an activity. Third, an inner harmony a person derives from engaging in an activity represents fulfillment of meaningfulness. The inner harmony results from the interaction of desire and striving. In this sense, inner harmony suggests a person's willingness to pursue the goal through continu-
ous engagement in the activity.

Research in physical education recently started to provide empirical evidence that supports this interpretation of the two constructs. Meanings in the content, specified by the purpose, intention arousal, and information in learning physical education, are often perceived differently by teachers and students. This inconsistency of perceived meaning in the content prevents students from gaining insights into meaningfulness that physical education can provide for their lives. As Lee and Solmon (1992) observed, students in physical education often impose their own ideas and thoughts on teachers' instructional directions to transform or internalize teachers' goals into something only meaningful to themselves.

In an examination of students' construction of personal meanings when learning physical skills, Langley (1995) found that, for various reasons, none of the four students sampled from a college bowling class demonstrated a personal goal compatible with the teacher's interpretation of the content purpose in learning the mechanics of hook ball. The data reported in Langley's study (1995) further indicated that the students did not acknowledge the meaning in bowling as the teacher 
expected. Similarly, Lee, Landin, and Carter (1992) reported that many students showed difficulties in recalling adequate information given by the teacher in a tennis unit. It was likely, the researchers speculated, that the incompatibility between the teacher's and students' goals in learning was due to their different perceptions of meanings in tennis. It can be argued that in a learning environment where the meaning of content is interpreted in a way that reveals little meaningfulness for students' lives, learning is likely to become a process of coping with, rather than acquiring, the knowledge and skills defined in the content (Langley, 1995).

Jewett and Bain (1987) explored meanings in physical education through their development of the Purpose-Process Curriculum Framework. From this perspective, meaning can be taught when students are moving through a seven-stage movement process to search for purposes, knowledge, and skills when engaged in physical activities. As Jewett and Bain (1987) described, meaning in physical activities is conceptualized as a three-dimensional construct of purpose, including self awareness, movement space, and social relations. The meanings, reflected in 23 content purposes in this framework, are defined for students to acquire.

Similarly, meanings, conceptualized as characteristics in various physical activities, were once categorized into six dimensions: social experience, fitness development, pursuit of vertigo (thrill pursuit), aesthetic experience, catharsis (tension release), and ascetic experience (self-control/discipline) (Kenyon, 1968a, 1968b). Although the construct was tested as valid and reliable for college students, little can be inferred regarding whether meaningfulness can be conceived of based on these meanings. In other words, little is known about whether or not persons' conceptions of meaningfulness in physical activities are consistent with their perceptions of meanings.

The two constructs were based on a general assumption that meaning can be internalized into meaningfulness by students when the purposes or characteristics in physical activities are revealed to them. With research evidence (e.g., Langley, 1995; Lee \& Solmon, 1992) suggesting that students may perceive meanings that are different from those of teachers, it seems reasonable to speculate that the two constructs overlooked the possibility that students may fail to internalize some of the meanings as meaningful, or may interpret them as undesirable. It may be assumed that students also differ in how they internalize meaning into meaningfulness. Differences in the conception of meaningfulness may lead students to either learn or avoid the physical education content when it is presented in class.

The above review of the literature showed that results from both theoretically oriented exploratory endeavors (e.g., Jewett \& Bain, 1987; Kenyon, 1968a, 1968b) and classroom-based research (e.g., Langley, 1995; Lee \& Solmon, 1992) suggest that educators must explore students' conceptions of meaningfulness to understand why students participate in physical education in the way they do. Gaining this understanding, in turn, may help teachers and curriculum workers in schools examine their curricula from a perspective meaningful to students. This study was designed to explore high school students' conceptions of meaningfulness in the physical education curriculum in terms of the theoretical framework of meaning and meaningfulness. This research purpose was expected to be accomplished by (a) eliciting perceived meanings in the physical activities, and (b) constructing and examining conceptions of meaningfulness derived from the meanings among high school students. 


\section{Method}

Procedurally, the study was carried out in two stages. First, a tentative construct of meaningfulness in physical education was elicited from a group of former high school students whose entire secondary education was completed in local public schools. They shared similar experiences in secondary physical education that was taught according to the same state curriculum guide. Second, an instrument measuring conceptions of meaningfulness, the Personal Meaningfulness Scale, was developed on the basis of this construct. The scale was administered to a sample of current high school students to construct their conceptions of meaningfulness. After the scale's internal consistency was established, students' responses to the items were analyzed in an attempt to reconstruct the meaningfulness construct and to test students' differentiation in conceptions of meaningfulness.

\section{Stage 1: Developing a Construct of Meaningfulness}

A construct of meaningfulness emerged from oral reflections of 35 former local high school students ( 17 female, 18 male; average age, 22) on their perceptions of meaning as related to physical activities perceived as interesting or boring (Chen, 1996, 1998). The students graduated from high school approximately 3 years ago. They were highly physically active, as judged by their records of regular enrollment in elective physical education courses in college, and reported daily participation in self-directed exercise. The physical activities they based their reflections on were those offered in the curriculum of their secondary schools. Reflections were audiorecorded, transcribed, and analyzed using an open-axial-selective comparison analysis (Strauss, 1987). Analyses of the data showed that the perceived meanings were structured around the students' perceptions of the purposes served, their intention elicited, and the knowledge or skills disseminated in physical education. Based on these meanings, a nine-dimensional meaningfulness construct was formed. The construct included socialization, social recognition, self-expression, activity variation, competence demonstration, competitiveness, cultural appreciation, tension release, and fitness development. The dimensions developed in the construct were regarded by the students as having a long-lasting and motivating effect on their continued participation in physical activities after their secondary education. The following is a brief description of the dimensions.

Fitness Development. This dimension emphasizes promotion of healthrelated fitness. Activities perceived as disseminating knowledge associated with personal health were considered relevant. Although the meaning in fitness activities was recognized by all students, only one-third indicated that they would invest their desire and effort to engage continuously in these activities. In other words, the meaning of fitness was well-perceived, but not all students internalized the meaning into meaningfulness.

Socialization. Socializing with old friends or making new friends were perceived as the purpose of engaging in physical education classes. Activities that help gain and use person-to-person interaction knowledge and skills were perceived as appropriate by the students. In a manner that was similar to their response to the meaning of fitness, only a subgroup of students internalized the meaning of socialization as meaningful to them.

Social Recognition. This dimension represents a desire to be recognized socially in the sport or exercise context. Knowledge, skills, and behavior in sport 
and exercise that can draw public attention were perceived as necessary to know. Desire, effort, and continuous pursuit in participating in an activity were perceived as dependent on whether the activity could provide a setting to use knowledge and skills to draw others' attention and admiration.

Activity Variation. This dimension reflects students' perceiving physical education as playing. Frequently alternating activities was expected by the students to keep a refreshed feeling when participating in physical activities. Knowledge and skills associated with different types of sports and activities were perceived as essential in this dimension. Playing a variety of activities in physical education was conceptualized as meaningful by a group of students who cared about being physically active more than learning knowledge and skills.

Self-Expression. In this dimension, expression of personal feelings and emotions derived from participating in certain activities, such as dance, is considered an ultimate purpose of physical education. The knowledge and skills about artistic expression, mainly used in dance and gymnastics events, were considered relevant by a small group of students in the sample. The components of meaningfulness, desire, striving, and continuous pursuit in these activities were expressed by the students who highly valued being able to communicate emotions to others.

Competitiveness. Winning is the sole purpose for participating in physical education. The knowledge and skills related to winning games in major regulation sports were considered to be important content. This meaning was conceptualized as meaningful by some students who emphasized extending the meaning of win and loss beyond the sport setting by implying the necessities of being competitive in daily life.

Competence Demonstration. This dimension represents a type of desire to demonstrate personal ability or competence in certain activities. Important knowledge and skills include those about engaging in a variety of physical activities, testing and comparing one's own ability either with one's previous levels or with others. Meaningfulness is characterized by being able to identify one's own abilities and competence. This meaningfulness was appreciated by the students for whom knowing about themselves and their ability determined what they should do.

Cultural Appreciation. The cultural appreciation dimension addresses the need for exploring and understanding various cultures represented in nonregulation sports or physical activities valued by local communities. Information about specific cultures was essential in an activity in which the students were expected to participate. The meaningfulness was observed among the students who reported a desire to continuously pursue the activities rooted in local multiethnic cultures.

Tension Release. This dimension emphasizes the function of stress reduction in physical education. Knowledge and skills perceived as useful in reducing stress were considered worth learning. Some students in the sample considered physical education meaningful to them only when it served as a means to help them reduce the tension or stress accumulated through intensive studies in other subject areas.

\section{Stage 2: Examination of Conceptions of Meaningfulness}

Context and Participants. Students in three high schools in the metropolitan area of Honolulu, Hawaii, served as the sample pool. Two of the schools, Midtown and King, were in the urban area, while the other, Palmtree, was in the suburb. Each school housed students in 9th, 10th, 11th, and 12th grades. The enrollment 
was 2,119 for Midtown, 2,048 for King, and 1,148 for Palmtree. The multicultural environment was represented by a mixed ethnic composition among students as well as teachers in each school. Although daily physical education was provided in all the schools, only 9th- and 10th-grade students were required to take it. For those in 11th and 12th grade, physical education was elective. The physical education programs in the three schools consisted of similar team sports and fitness development concentrations. Regulation sports were central to the curricula across schools. Fitness development was incorporated in the programs in different ways. Students in Midtown and King spent first 10 to 20 minutes of each class on fitness development activities before they were given instruction in sport areas. At Palmtree, however, every Wednesday was designated as a fitness day on which the entire physical education period was devoted to fitness development activities.

A total of 698 students (52\% male, $48 \%$ female) from the three schools participated in the study. In Midtown and King, three classes were randomly selected from each of the 9th, 10th, and 11th grades, while two classes were selected from each of those grades in Palmtree. Students sampled in each school represented approximately $11 \%$ to $12 \%$ of the school population. These students were taught by seven physical education specialists with teaching experiences ranging from 11 to 32 years. Two teachers were Caucasian female, two Caucasian male, one African American male, and two males had Asian-Pacific ethnicity.

Scale Development. A Personal Meaningfulness Scale was developed for this study based on the analyzed oral reflection data from which the nine-dimensional construct of meaningfulness was derived. A total of 61 oral statements from the students' reflection data were selected as stem sentences to represent the dimensions. These sentences reflected the students' conceptions of meaningfulness in that the three components of the construct, desire, striving, and inner harmony, could be identified. During scale development, each sentence was edited to change it from its colloquial form into a written format with a complete sentence structure. Additional editing was performed to (a) add an "I" at the beginning of an item if it was omitted in the original sentence, (b) change the past tense of the verb, if any, to the present tense, and (c) change the passive voice of the verb, if any, to the active voice. The 61 items were randomly placed in the scale. Five foil items were written and also randomly placed in the scale. The foil items stated commonly-known incorrect facts that could be identified immediately by a rater who was carefully completing the scale. The foil items served the purpose of identifying those respondents who did not complete the scale carefully. A 5-point Likert-type rating scale was applied to each item polarized with the descriptors of "Very Untrue" and "Very True." Table 1 lists a few items in the scale, including a foil item.

Data Collection. The scale was distributed to the students in their classrooms with a consent form. The students were informed of their right to choose not to participate prior to the distribution of the scale and assured of anonymity of their responses. Participation was considered voluntary when the consent form was signed. The students were also informed that participation in the study would not affect their grades in physical education. All the scales were distributed to, rated by, and collected from the students within one class period. The teacher who was teaching the class helped in scale distribution and collection. Data collection in different classes and schools followed an identical protocol: (a) announcement of the purpose, the right not to participate, and the noninterference principle (ad- 
Table 1 Sample Items in Personal Meaning Inventory

\begin{tabular}{|c|c|c|c|c|}
\hline Statements & $\begin{array}{c}\text { Very } \\
\text { untrue }\end{array}$ & $\begin{array}{l}\text { Not } \\
\text { sure }\end{array}$ & & $\begin{array}{l}\text { ery } \\
\text { ue }\end{array}$ \\
\hline 6. I want to play the sports that my boy/girl friend likes. & & 34 & 4 & 5 \\
\hline 17. I want to show my friends my talent in sport classes. & 12 & 34 & 4 & 5 \\
\hline 28. I participate in physical activity to control my weight. & 12 & 34 & 4 & 5 \\
\hline 45. I always want to be challenged in physical education. & 12 & 34 & 4 & 5 \\
\hline Foil: Heavy snow makes Honolulu a first-class ski resort. & 12 & 34 & 4 & 5 \\
\hline
\end{tabular}

dressing all questions to the researcher by raising hands), (b) scale distribution, (c) scale collection, (d) dismissal of the class.

Data Analysis. A preliminary screening of completed scales was performed first. The scales completed with a single rating (e.g., 5 assigned to all items) were eliminated from further analysis. The preliminary screening was followed by a descriptive analysis of responses on foil items to determine the overall trustworthiness of the responses. Those completed scales with a mean higher than 2 for the five foil items were identified and eliminated from further analysis because a mean greater than 2 indicated that the student did not rate all the items with the same degree of carefulness and attention. An analysis of internal consistency (Cronbach's $\alpha$ ) was then performed to identify and delete items that affected internal consistency of the scale. Thus, only those items that carried reliable responses from the students were entered into the subsequent analyses. Carrying out this procedure first was considered necessary, because the items derived directly from the oral reflection data and their internal consistency (ability to elicit reliable responses) was unknown otherwise.

After the internal consistency of the scale was substantiated, students' responses to the remaining items were reduced using the principal component method to reconstruct the dimensions of meaningfulness. The initial construct elicited from the exploratory analysis was tested using confirmatory factor analysis (CFA) to verify the extent to which the construct of meaningfulness fitted the data. Modification of the construct was made in terms of the diagnosis solutions obtained in CFA. Then, a multivariate analysis of variance (MANOVA) was used to examine differentiation of means of students' responses to the valid dimensions of meaningfulness (aggregated scores on Social Bonding, Culture Appreciation, Challenge, Tension Release, Fitness Development, and Self-Expression) in terms of their grade, gender, and socioeconomic status.

\section{Results}

\section{Item Internal Consistency}

The preliminary screening revealed that 74 completed scales failed to meet the screening criteria. They were then dropped from the sample for further analyses. The screening resulted in a sample of 624 students $(89.4 \%$ of the total). Their demographic data are reported in Table 2. 
Table 2 Demographic Data of the Students $(N=624)$

\begin{tabular}{|c|c|c|c|c|}
\hline & $\begin{array}{c}\text { Midtown } \\
(N=250) \\
n(\%)\end{array}$ & $\begin{array}{c}\text { King } \\
(N=226) \\
n(\%)\end{array}$ & $\begin{array}{c}\text { Palmtree } \\
(N=148) \\
n(\%)\end{array}$ & $\begin{array}{l}\text { Total } \\
n(\%)\end{array}$ \\
\hline \multicolumn{5}{|l|}{ Gender } \\
\hline Male & $145(58.0)$ & $122(54.0)$ & $88(59.5)$ & 355 (56.9) \\
\hline Female & 104 (41.6) & $101(44.7)$ & $57(38.5)$ & $262(42.0)$ \\
\hline Missing data & $1(0.4)$ & $3(1.3)$ & $3(2.0)$ & 7 (1.1) \\
\hline \multicolumn{5}{|l|}{ Grade } \\
\hline 9th & $75(30.0)$ & 94 (41.6) & $50(33.8)$ & $219(35.1)$ \\
\hline 10th & $102(40.8)$ & $64(28.3)$ & $53(35.8)$ & $219(35.1)$ \\
\hline 11th & $72(28.8)$ & $65(28.8)$ & $42(28.4)$ & $179(28.7)$ \\
\hline Missing data & $1(0.4)$ & $3(1.3)$ & $3(2.0)$ & 7 (1.1) \\
\hline \multicolumn{5}{|l|}{ Lunch } \\
\hline Free/reduced & $72(28.8)$ & $55(24.3)$ & $2(1.4)$ & $129(20.7)$ \\
\hline Own & $156(62.4)$ & $146(64.6)$ & $134(90.5)$ & 436 (69.9) \\
\hline Missing data & $22(8.8)$ & 25 (11.1) & $12(8.1)$ & $59(9.4)$ \\
\hline \multicolumn{5}{|l|}{ Ethnicity } \\
\hline African & $5(2.0)$ & $4(1.8)$ & $1(0.7)$ & 10 (1.6) \\
\hline Asian & $40(16.0)$ & $28(12.4)$ & $35(23.6)$ & $103(16.5)$ \\
\hline Caucasian & $14(5.6)$ & $3(1.3)$ & $18(12.2)$ & $35 \quad(5.6)$ \\
\hline Filipino & $103(41.2)$ & 93 (41.2) & $8(5.4)$ & $204(32.7)$ \\
\hline Hawaiian & $17(6.8)$ & $14 \quad(6.2)$ & $3(2.0)$ & $34(5.5)$ \\
\hline Samoan & $23(9.2)$ & $43(19.0)$ & $68(45.9)$ & $134(21.5)$ \\
\hline Mixed & $43(17.2)$ & 33 (14.6) & $9(6.1)$ & $85(13.6)$ \\
\hline Missing data & $5(2.0)$ & $8(3.5)$ & $6(4.1)$ & $19(3.0)$ \\
\hline
\end{tabular}

To minimize the chance of producing an unreliable construct, Cronbach's (1951) approach was used prior to the principal component analysis to identify and prevent the items with low internal consistency from entering the principal component analysis. During the analysis, items that weakened internal consistency of a dimension were eliminated to improve the internal consistency of the scale. The dimension of Activity Variation was dropped because of its overall low item internal consistency $(\alpha=.44)$. As reported in Table 3, Cronbach's $\alpha$ for the subscales ranged from .61 to .71 . Considering the number of items in each subscale, the moderate internal consistency coefficients were considered acceptable. The item internal consistency test and the item elimination process yielded a total of 39 items in eight dimensions for the entire scale.

\section{Principal Component Analysis}

The principal component analysis was conducted to reduce students' responses to the 39 items into a construct of meaningfulness. Bartlett's test of sphericity showed that the correlation matrix of the 39 items was a nonidentity matrix (Bartlett Sphericity $=1387.46, p<.01$ ), indicating that the data met the primary 
Table 3 Item Internal Consistency of Personal Meaningfulness Scale

\begin{tabular}{lcccc}
\hline & $\begin{array}{c}\text { Total } \\
\text { number of } \\
\text { Dimensional } \\
\text { subscale }\end{array}$ & $\begin{array}{c}\text { Number of } \\
\text { items } \\
\text { remaining }\end{array}$ & $\begin{array}{c}M / S D \\
(N=624)\end{array}$ & $\begin{array}{c}\text { Cronbach's } \\
\alpha\end{array}$ \\
\hline Socialization & 8 & 6 & $3.45 / .76$ & .68 \\
Social recognition & 6 & 4 & $3.18 / .57$ & .69 \\
Self-expression & 7 & 5 & $3.34 / .66$ & .63 \\
Activity variation ${ }^{a}$ & 7 & 0 & $2.87 / .37$ & .44 \\
Fitness development & 6 & 4 & $3.47 / .65$ & .61 \\
Cultural appreciation & 7 & 5 & $3.18 / .68$ & .70 \\
Tension release & 7 & 5 & $3.33 / .54$ & .64 \\
Competence demonstration & 6 & 5 & $3.39 / .69$ & .71 \\
Competitiveness & 7 & 5 & $3.42 / .61$ & .68 \\
& & & & \\
\hline
\end{tabular}

${ }^{a}$ This subscale was dropped from the following factor-analysis because of the low internal consistency coefficient $(\alpha=.44)$.

assumption for generating a valid and reliable factor solution. The conventional criterion, Eigenvalue = 1 (Kaiser, 1974), was used to determine extraction of factors. With compliance to this criterion, eight factors were extracted initially, as reported in Table 4. The variance accounted for by this eight-factor model was $70.5 \%$. Based on Guadagnoli and Velicer's (1988, as cited in Stevens, 1992) recommendation that only those factors with at least four high-loading values (.40$.60)$ be considered valid and reliable, two factors (Factor 7 and 8 in Table 4) were dropped from the construct. The remaining six factors formulated a six-dimensional construct of meaningfulness for this sample of students. This model accounted for $65 \%$ in variance in which Social Bonding, Culture Appreciation, Challenge, Tension Release, Fitness Development, and Self-Expression accounted individually for $23.4 \%, 16.4 \%, 9.2 \%, 7.7 \%, 5.2 \%$, and $3.1 \%$, respectively.

An examination of the items reduced into the six dimensions revealed that an item integration occurred during the factor analysis. Table 5 contrasts the new dimensions with their sources. It was found that items originally in Socialization, Social Recognition, Culture Appreciation, and Self-Expression were reorganized in the factor analysis, forming new dimensions of Social Bonding, Culture Appreciation, and Self-Expression. A new dimension of Challenge consisted of items from original dimensions of Competitiveness and Competence Demonstration. However, the item reorganization was not observed in the Fitness Development and Tension Release dimensions. The name of each dimension was determined subjectively, based on the researcher's understanding of the theme reflected in the items in each dimension.

\section{Confirmatory Factor Analyses}

Subsequently, the confirmatory factor analysis was used to verify the meaningfulness construct. In this case, indicators introduced in the analysis paths were 
Table 4 Factor Loading on Meaingfulness Dimensions Identified in Factor Analysis

\begin{tabular}{|c|c|c|c|c|c|c|c|c|}
\hline Item & $\begin{array}{l}\text { Factor } 1 \\
\text { Social } \\
\text { bonding }\end{array}$ & $\begin{array}{c}\text { Factor } 2 \\
\text { Cultural } \\
\text { appreciation }\end{array}$ & $\begin{array}{l}\text { Factor } 3 \\
\text { Challenge }\end{array}$ & $\begin{array}{l}\text { Factor } 4 \\
\text { Tension } \\
\text { release }\end{array}$ & $\begin{array}{c}\text { Factor } 5 \\
\text { Fitness } \\
\text { development }\end{array}$ & $\begin{array}{c}\text { Factor } 6 \\
\text { Self- } \\
\text { expression }\end{array}$ & $\begin{array}{l}\text { Factor } 7 \\
\text { (Invalid) }\end{array}$ & $\begin{array}{l}\text { Factor } 8 \\
\text { (Invalid) }\end{array}$ \\
\hline Item 61 & .77140 & .06443 & -.00484 & .05349 & -.00572 & .02753 & .14033 & -.07933 \\
\hline Item 6 & .72600 & .10880 & -.04183 & .02983 & .08779 & .08505 & .14131 & -.05467 \\
\hline Item 63 & .54707 & .11571 & .26204 & .19888 & .15890 & -.03685 & -.04425 & .09094 \\
\hline Item 29 & .52102 & .08352 & .24182 & .00670 & .01186 & .15401 & .00251 & 21377 \\
\hline Item 19 & .49321 & .16561 & .24230 & -.12362 & .16157 & .14853 & .17223 & .15997 \\
\hline Item 43 & .47592 & .17708 & 15903 & .02164 & -.04843 & .20753 & -.04952 & .15378 \\
\hline Item 44 & .45396 & .31851 & .10367 & .42546 & -.14511 & .05931 & -.00611 & -.14129 \\
\hline Item 27 & .43637 & .08757 & .26469 & -.00332 & .18756 & .20801 & -.04375 & .26435 \\
\hline Item 42 & 10716 & .70278 & .13503 & .16276 & .04674 & .02217 & .15742 & .10300 \\
\hline Item 49 & .04395 & .66809 & .18133 & .14923 & .20010 & .06166 & .09737 & .03957 \\
\hline Item 25 & .12417 & .55006 & -.07051 & .20077 & .22435 & .16812 & .18552 & .21639 \\
\hline Item 31 & .15370 & .54420 & .24858 & .06334 & -.09981 & .23682 & -.03663 & .19510 \\
\hline Item 7 & .21053 & .53687 & .04947 & .06509 & .09131 & .19363 & .11700 & .10060 \\
\hline Item 41 & .18336 & .50623 & .03701 & -.06649 & .19611 & .00369 & -.33096 & .06550 \\
\hline Item 46 & .09489 & .41048 & .28025 & .33931 & .36015 & .00717 & .08905 & -.13845 \\
\hline Item 36 & .17363 & .19336 & .54620 & -.12466 & .03820 & .26291 & .18277 & .04764 \\
\hline Item 56 & .15352 & .19207 & .52938 & .24087 & .11430 & .05854 & .04910 & .03439 \\
\hline
\end{tabular}




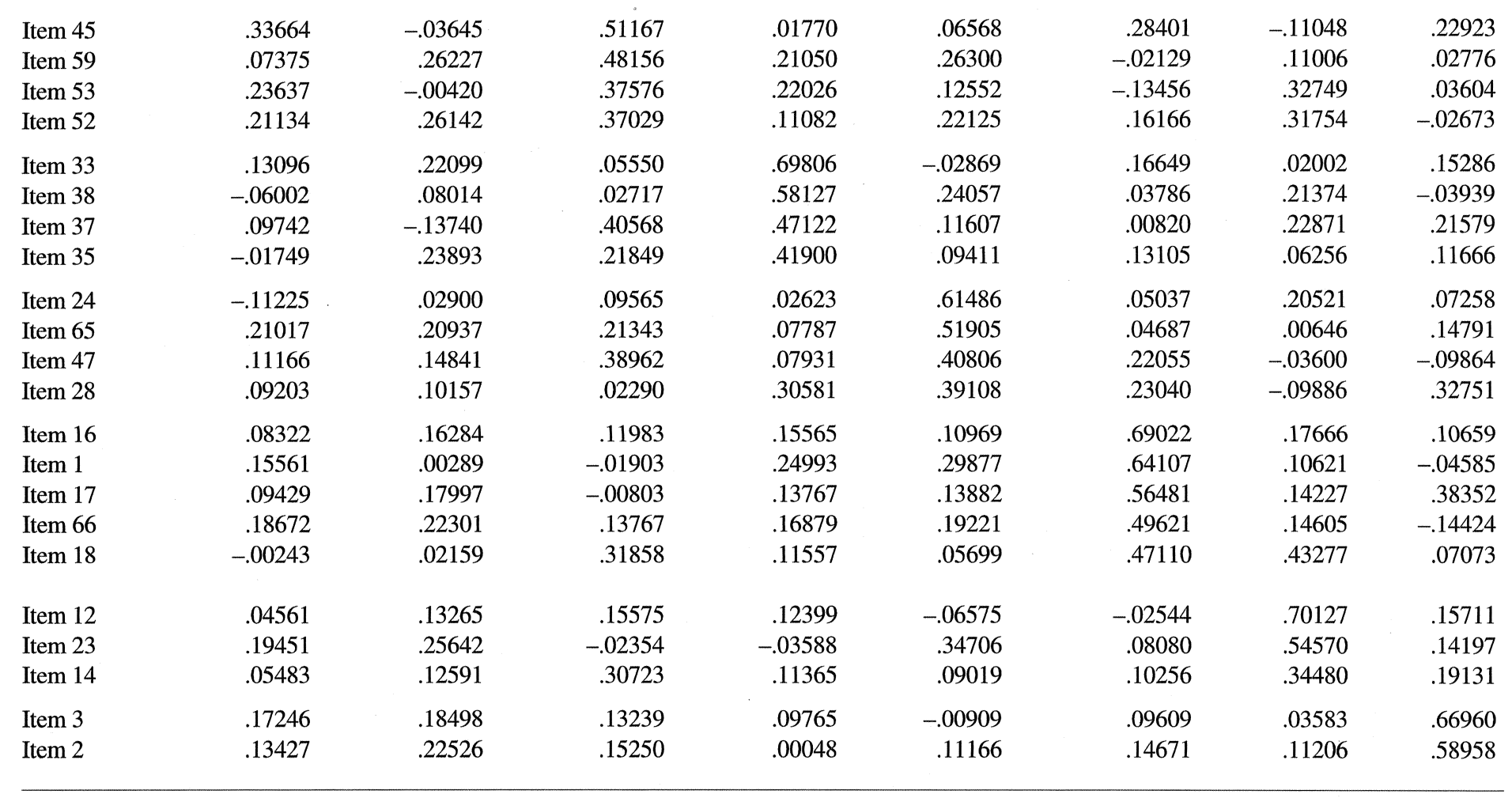


Table 5 Dimensions Integrated and the Sources

\begin{tabular}{|c|c|c|c|}
\hline New dimension & $\begin{array}{l}\text { Number } \\
\text { of items }\end{array}$ & $\begin{array}{l}\text { Source } \\
\text { dimension }\end{array}$ & $\begin{array}{l}\text { Number } \\
\text { of items }\end{array}$ \\
\hline Social bonding & 8 & Socialization/social recognition & $6 / 4$ \\
\hline Cultural appreciation & 7 & $\begin{array}{l}\text { Cultural appreciation/socialization/ } \\
\text { self-expression }\end{array}$ & $5 / 5 / 6$ \\
\hline Challenge & 6 & $\begin{array}{l}\text { Competitiveness/competence } \\
\text { demonstration }\end{array}$ & $5 / 5$ \\
\hline Tension release & 4 & Tension release & 4 \\
\hline Fitness development & 4 & Fitness development & 4 \\
\hline Self-expression & 5 & $\begin{array}{l}\text { Self-expression/cultural appreciation/ } \\
\text { socialization }\end{array}$ & $5 / 5 / 4$ \\
\hline
\end{tabular}

students' rating scores on the 39 items. The latent variables were the six dimensions of elicited meaningfulness. The analyses were computed using the LISREL ${ }^{\circledR}$ 8.0 program (Joreskog \& Sorbom, 1993). Covariance matrices of the items, which were generated using the PRELIS ${ }^{\circledR}$ program associated with the LISREL ${ }^{\circledR} 8.0$ program, served as the preliminary data base in the confirmatory factor analysis. The maximum likelihood solution was chosen as the analysis approach, because the objective of analysis at this stage was to examine the extent of likelihood that the model fitted the data. In other words, it was tested whether the differentiated conceptions of meaningfulness in this student sample had generated the observed responses in their ratings on the selected items.

The six-dimension meaningfulness construct was examined in three steps: (a) examining parameter estimates $\left(R^{2}\right)$ to see if the dimension-item relationships in the construct were sound; (b) examining the goodness-of-fit of the model; and (c) following the diagnostic suggestions given in the modification indices to modify the construct. The first run of the analysis revealed that the items demonstrated satisfactory likelihood loadings on their parent dimensions with individually explained variances ranging from .14 to .49 , suggesting that the relationships within the construct were generally sound.

The goodness-of-fit was judged based on Chi-square $\left(\chi^{2}\right)$, Goodness-of-Fit Index (GFI) and Adjusted Goodness-of-Fit Index (AGFI), and standard residuals of individual items. The $\chi^{2}(d f=480)$ generated from the analysis was $729(p<.001)$, indicating that the construct fitted the data poorly. However, because the Chi-square analysis is a sample-size sensitive approach (it tends to produce large $\chi^{2}$ when the sample size is large), it has been recommended (Joreskog \& Sorbom, 1993) that other indices should be used in the cases involving large sample sizes. Therefore, GFI, AGFI, and standard residuals, which are independent from effects of sample sizes, were examined by the researcher to gather additional information for evaluating the construct. The GFI and AGFI were .89 and .86 , respectively, suggesting the construct almost reached the commonly acceptable criterion for a fit (.90). The standard residuals from the items were scattered around the base point 0 orderly, and their values ranged from 0 to 3.8 on both positive $(+)$ and negative $(-)$ directions, indicating absences of model/data violations, also a sign of an acceptable fit. 
The examination of model modification indices showed that several items had shared paths between two or three dimensions. These items and their original and shared paths were reported in Table 6 . Normally, adding these paths to the dimensions would decrease the $\chi^{2}$ value, consequently, a non-significant $\chi^{2}$ could be obtained. However, the statistical solution (addition of shared paths in this case) must be justifiable based on substantive and theoretical reasons. After a thorough examination of the suggested target dimensions (shared paths) and the multipath items, it became clear that the additions would hardly be justified substantively based on the descriptions of the meaningfulness dimensions. The decision to eliminate these multipath items from their original dimensions was made by the researcher in an effort to adjust the construct to achieve the criterion for fitting the data.

The subsequent repetition of the CFA analyses on the remaining items showed that the $R^{2}$ remained at the acceptable level, ranging from .11 to .44 as reported in Table 7. As a result of the deletion, the GFI and AGFI increased to .91 and .89 , respectively. Similar standard residuals were observed. Although the deletion decreased the $\chi^{2}$ value, it was not able to bring it down to a statistical nonsignificance level (it was not expected to because of the sample-size sensitivity). The analyses resulted in a construct of meaningfulness represented by the items listed in the appendix.

Effects of the socioeconomic status, gender, and grade on students' conceptions of meaningfulness were examined using multivariate analysis of variance (MANOVA). The data from the three schools were pooled for the MANOVA because no school effect was found in a one-way analysis of variance (ANOVA) that preceded the MANOVA. The MANOVA analyses revealed no interaction effects from the socioeconomic status (Free/Reduced/Own Lunch), gender, and grade factors. However, as reported in Table 8, each of these factors independently had a sig-

Table 6 Multipath (Dimension) Items Diagnosed Confirmatory Factor Analysis

Item

Original/shared path (dimension)

27. I want my friends to watch me playing sports that I am good at.

44. I want to be able to play my family sports.

63. I enjoy playing those sports that my friends think are "cool stuff."

41. I enjoy sports that have cultural meanings for the movements.

46. I want to learn those sports that help me understand other peoples.

36. I want to learn those sports that I can't play well.

35. I relax in PE to release tension from study in the classroom.

18. I enjoy practicing sports that show the gracefulness of my movement.
Social bonding/challenge

Social bonding/self-expression

Social bonding/fitness development

Cultural appreciation/fitness

Cultural appreciation/tension release

Challenge/tension release

Tension release/cultural appreciation

Self-expression/challenge 
Table 7 Variance Explained by Individual Item Within Each Dimension

Dimension

Item \# $R^{2}$

\begin{tabular}{lrrrr} 
Social bonding & $6 / .43$ & $19 / .25$ & $29 / .21$ & $43 / .11$ \\
Cultural appreciation & $7 / .27$ & $25 / .33$ & $42 / .20$ & $49 / .31$ \\
Challenge & $45 / .28$ & $52 / .14$ & $53 / .39$ & $56 / .25$ \\
Tension release & $33 / .17$ & $37 / .33$ & $38 / .26$ & \\
Fitness development & $24 / .22$ & $28 / .22$ & $47 / .20$ & $65 / .13$ \\
Self-expression & $1 / .44$ & $16 / .25$ & $17 / .35$ & $66 / .12$ \\
\hline
\end{tabular}

nificant impact on differentiating the students' conceptions of meaningfulness ( $p=$ .025 for Free/Reduced/Own Lunch, $p=.001$ for Gender, $p=.001$ for Grade). Power analysis results (Power $=.79, .98, .99$ for Free/Reduced/Own Lunch, Gender, and Grade, respectively) suggest that the rejections to the hypotheses set for MANOVA (there were no differences caused by the three factorial variables) were sound. Results of the univariate analyses on the factorial variables of Lunch, Gender, and Grade, as reported in Tables 9 and 10, showed that the students in the Free/Reduced Lunch programs rated Social Bonding and Cultural Appreciation higher than other students $(p<.05)$. Male students highlighted Social Bonding, Cultural Appreciation, and Chaladdition, students in female counterparts $(p<.01, p<.05, p<.01$, respectively). In higher than their peers in the lower grades.

\section{Discussion}

In this study, high school students' conception of meaningfulness in physical education was examined within a framework that assumes that the conception of meaningfulness derives but is independent from the perception of meaning in the content. The examination was accomplished by (a) eliciting a construct of meaningfulness based on former high school students' perceptions of meanings in physical education, (b) testing the construct among current high school students, and (c) examining differentiation of the students' conceptions of meaningfulness
in terms of their gender, grade, and socioeconomic status.

\section{Meaningfulness: A Sophisticated Construct}

The formation of the nine dimensions of meaningfulness from the reflection data implies that the students conceptualized meaningfulness in physical education through internalizing meanings perceived in physical activities. However, the nine dimensions in this hypothesized construct were reconstructed into six dimensions in the analyses, indicating an inconsistency between the two constructs. The change reflects the sophistication of conceptualizing meaningfulness in physical
activities. The data analyzed in the study may offer three interrelated interpretations for the reconstruction of the constict. differentiation in conceptualizing meanstruct. First, the reconstruction reflected a dents who highly valued regular participatiness between former high school stu- 
Table 8 Results of MANOVA on Effects of Grade, Gender, and Socioeconomic Status (Lunch)

\begin{tabular}{|c|c|c|c|c|c|c|}
\hline Effect & Value $^{a}$ & $\begin{array}{l}\text { Hypothesis } \\
\qquad d f\end{array}$ & $\begin{array}{l}\text { Error } \\
\quad d f\end{array}$ & $F$ & $p$ & $\begin{array}{c}\text { Observed } \\
\text { power }^{b}\end{array}$ \\
\hline \multicolumn{7}{|l|}{ Interaction effects } \\
\hline Lunch/gender/grade & .96 & 12.00 & 1052.00 & .36 & .18 & .63 \\
\hline Gender/grade & .98 & 12.00 & 1052.00 & .96 & .49 & .57 \\
\hline Lunch/grade & .97 & 12.00 & 1052.00 & .97 & .17 & .77 \\
\hline Lunch/gender & .99 & 6.00 & 526.00 & .99 & .79 & .21 \\
\hline \multicolumn{7}{|l|}{ Main effect } \\
\hline Grade & .93 & 12.00 & 1052.00 & 3.27 & .001 & .99 \\
\hline Gender & .05 & 6.00 & 526.00 & 4.50 & .001 & .98 \\
\hline Lunch & .03 & 6.00 & 526.00 & 2.23 & .025 & .79 \\
\hline
\end{tabular}

${ }^{a}$ For interaction effects and Grade main effect, the value $=$ Wilk's $\lambda$; for Gender and Lunch main effects, the value $=$ Hotelling's $T^{2}$.

${ }^{b}$ Percentage of chances to reject the null hypothesis correctly when the criterion is set at .05 .

sample representing a current high school population that might value physical activities to a lesser degree. Given the discrepancy in the two samples, it may be speculated that the former high school students might have developed a system or systems of perceiving meanings and conceptualizing meaningfulness. Their system can be more complex and sophisticated than those of current high school students, encompassing a broad spectrum of meaningfulness dimensions.

A second interpretation of the change could be that current high school students have not developed such a system that enables them to discriminate and internalize the subtleties between or among similar meanings. The interpretation is partially supported by the evidence that integration occurred among the similar affective meaningfulness dimensions. Items related to the four affective dimensions, Socialization, Social Recognition, Self-Expression, and Cultural Appreciation, were regrouped and integrated in both principal component and confirmatory factor analyses. Integration of the dimensions (see Table 5) may well indicate that the current high school students had difficulties in distinguishing the subtle meanings among these similar dimensions related to affective meanings in physical activities. In addition, the elimination of the Activity Variation dimension may support this notion as well. All items in this dimension were rated overwhelmingly low by current high school students $(M=2.87, S D=.37)$, indicating that the high school students did not recognize that varying activities may be a meaningful way to motivate themselves to continue participation in physical activities, as did the former students.

For those former high school students, on the other hand, meaningfulness became highly meaning- and activity-specific. It is likely that their judgment on meaningfulness depended, in part, upon their postsecondary experiences obtained through their regular, persistent participation in certain physical activities (Chen, 1996). Their conceptions of meaningfulness, therefore, might have reached a level 
Table 9 Results of Univariate Analysis on Effects of Grade, Gender, and Socioeconomic Status (Lunch)

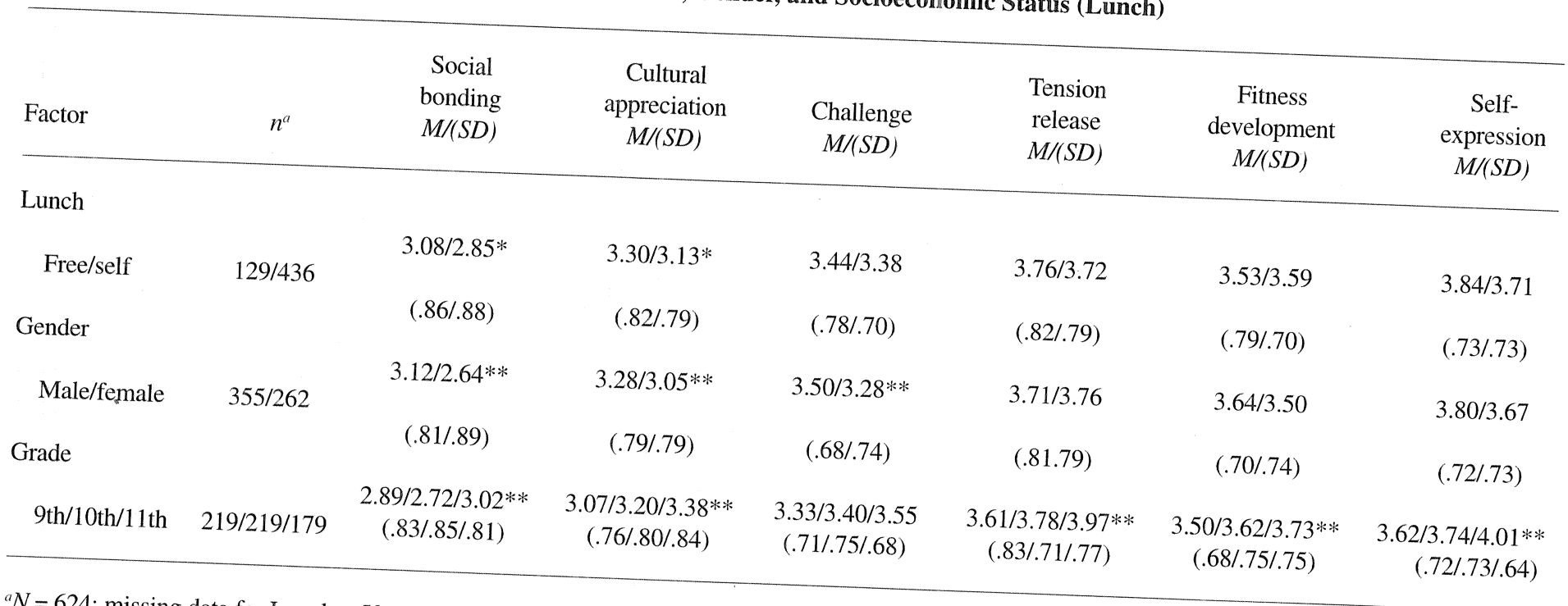

${ }^{a} N=624 ;$ missing data for $\operatorname{Lunch}=59 ;$ Gender $=7 ;$ and Grade $=7$.
${ }^{*} p<.05 ; * *<<.01$. 
Table 10 Post-Hoc Analysis on Mean by Grade

\begin{tabular}{|c|c|c|c|c|c|c|c|c|c|c|c|c|}
\hline & \multicolumn{2}{|c|}{$\begin{array}{c}\text { Social } \\
\text { bonding }\end{array}$} & \multicolumn{2}{|c|}{$\begin{array}{c}\text { Cultural } \\
\text { appreciation }\end{array}$} & \multicolumn{2}{|c|}{ Challenge } & \multicolumn{2}{|c|}{$\begin{array}{l}\text { Tension } \\
\text { release }\end{array}$} & \multicolumn{2}{|c|}{$\begin{array}{c}\text { Fitness } \\
\text { development }\end{array}$} & \multicolumn{2}{|c|}{$\begin{array}{c}\text { Self- } \\
\text { expression }\end{array}$} \\
\hline & 9th & 10th & 9 th & 10th & 9th & 10 th & 9th & 10th & 9 th & 10 th & 9 th & 10th \\
\hline 10th & - & - & - & - & - & - & - & - & - & - & - & - \\
\hline 11th & $p<.05$ & $p<.05$ & $p<.05$ & - & $p<.05$ & - & $p<.05$ & - & $p<.05$ & - & $p<.05$ & $p<.05$ \\
\hline
\end{tabular}

Note. The symbol - denotes no significant difference between the pair of means. 
of sophistication more complex than that of current high school students who were yet to develop this type of sophistication of conceptions. In other words, the reconstruction of the dimensions observed in this study may suggest a strong ability among those who valued physical activity participation to discriminate various meanings in physical activities in order to conceptualize the meaningfulness.

The third interpretation relates to research methodology. As compared with the statistical approach used in this study, the constant comparison approach (a naturalistic analytical method), used to elicit the hypothesized nine-dimensional construct, enables researchers to reach the subtlety, richness, and sophistication embedded in qualitative data (Goetz \& LeCompte, 1984). Although the statistical analyses generated useful findings that may be generalizable to a certain degree, it may be the case that the subtlety and sophistication of the meaningfulness detected in the former high school students' data were likely to be suppressed in the statistical analyses. Readers are advised to take the methodological issue into account when attempting to interpret the reported six-dimensional construct in relation to its hypothesized nine-dimensional counterpart.

In summary, the analyses for reconstructing the meaningfulness construct revealed a six-dimensional model. Given the changes in the dimensions from the original, hypothesized construct, the six-dimensional construct may be considered a preliminary model portraying high school students' conceptions of meaningfulness in participating in physical education. Research is needed to confirm the above speculative interpretations. It is also worthwhile to explore a hypothesis, which derived from the above analyses, that the conception of meaningfulness would develop into a sophisticated structure when the understanding of meanings in physi-
cal activities is enhanced.

\section{Differentiation of the Meaningfulness}

Although the students acknowledged the meanings in physical activities and were able to formulate the meaningfulness in terms of their perceptions of personal relevancy, their conceptions of meaningfulness were differentiated based on their socioeconomic status, gender, and grade levels. The MANOVA analysis revealed that students in the school-assisted lunch programs demonstrated a stronger desire than others to seek social affiliation opportunities and a higher intention to engage in activities centered on local cultures in physical education. Students with low socioeconomic status were often found in a state of social deficits. As Bye and Jussim (1993) have found, they are likely to be in need of social intelligence, social competence, and social knowledge. The higher ratings on affective meaningfulness dimensions by them may imply that the students are not only aware of the need but also able to seek and internalize the affective meanings in physical
education.

Conceptions of meaningfulness were also found to differentiate in several dimensions based on gender. Male students produced higher response scores in Social Bonding, Cultural Appreciation, and Challenge dimensions than the female students. The differentiation demonstrated that male and female students not only had different patterns of participation (Griffin, 1984, 1985) and perceptions of the gymnasium learning environment (Lirgg, 1994), but also differed in conceptions of certain meaningfulness dimensions in physical education. Although further speculation of the influence of these demographic variables on the conception of meaningfulness is inappropriate 
given the limitation in the data collected, the differentiation detected in the data certainly warrants further investigation on these issues.

In addition, students' conceptions of meaningfulness differed in terms of their grade levels. The results of post-hoc analysis (Table 10) seem to indicate that the more years of physical education students received, the more likely they demonstrated a strong conception of meaningfulness. The fact that the 11 th-grade students selected physical education and demonstrated a higher appreciation of meaningfulness across the dimensions may indicate, to the least extent, that the mandatory physical education in the 9 th and 10 th grades presented them with a positive and meaningful experience. The results revealed in this part of the data did reflect a positive impact of physical education on conceptions of meaningfulness.

The differentiation observed in the MANOVA analysis lends support to the notion that the conception of meaningfulness is individually constructed. The conception seemed to follow a process of construction that varies from person to person in terms of personal experiences in life and is sequenced in terms of years of physical education received. Csikszentmihalyi (1990) highlighted the process with three stages. At the first stage, individuals strive to preserve the self by exploring who they really are and what they want to achieve in life. When a person has little doubt about the self, he or she "may expand the horizon of his or her meaning system to embrace the values of a community. . . . This step leads to a greater complex of the self" (Csikszentmihalyi, 1990, p. 221). The second stage involves the development of a reflective individualism. The person integrates the community values into the self, internalizing meanings perceived relevant into an "autonomous conscience" (Csikszentmihalyi, 1990, p. 221). This conscience leads to the third stage, where the person develops the desire, striving, and continuous pursuit for relevant meanings. It is at this stage that the person willingly invests energy to seek opportunities to participate in activities that offer the relevant meanings. It is at this stage that meaningfulness is conceptualized and the conception is demonstrated through a person's action.

The differentiation observed in the students' data may suggest that they were at different stages of developing their conceptions of meaningfulness. This process of constructing meaningfulness most likely is influenced by students' demographic backgrounds, learning experiences, and social milieu. Thus, physical educators should feel neither surprised nor afraid when they observe students demonstrating different attitudes, interests, and behaviors in physical education classes. Instead, these differentiations should be viewed as the representations of their actions to search for and internalize meanings in physical education. Based on the framework that distinguishes meaning and meaningfulness, it may be hypothesized that students perceive meanings in physical activities as either meaningful or meaningless. It can be further assumed that their conceptions of meaningfulness or meaninglessness may depend primarily on how physical educators reveal and emphasize meaning in the physical activity to be taught.

\section{Implications for Curriculum Innovation}

The six-dimensional construct of meaningfulness indicates that students' conceptions of meaningfulness in physical education are characterized by pluralism. Pluralism is recognized as one of the salient characteristics of the postmodern era, which some scholars argue our society entered in the early 1970s. Unfortunately, our school curricula have demonstrated a strong reluctance to engage in pluralism (Doll, 1992; 
Slattery, 1995). Nonetheless, pluralism has been manifested both conceptually and empirically in education and physical education. Conceptually, postmodernist curriculum theories have been proposed to emphasize diversified approaches to curriculum development that encompass scientific, aesthetic, and constructivist paradigms (Doll, 1992; Kirk, 1993) and a student-centered, autobiographical approach to teaching (Slattery, 1995). The primary goal of the curriculum, then, should be reconceptualized as many processes in which teachers help students uncover, interpret, and internalize meanings in the content. Empirically, pluralism has been observed in teachers' educational value orientations (Ennis, 1992), students' differentiated perceptions of meanings (Langley, 1995; Lee et al., 1992), and their perceptions of interests and boredom in physical activities (Chen, 1996, 1998).

Developing a curriculum that is meaningful to the student requires curriculum workers and teachers to accept pluralism as a fundamental principle. Based on this principle, educators should acknowledge students' pluralistic interpretations and internalizations of meaning in the content. Results of this study seem to echo the literature, suggesting that curriculum workers and teachers should emphasize meanings significant to students' lives. In the meantime, they also need to facilitate students' internalization of meanings to develop the "autonomous conscience" (Csikszentmihalyi, 1990, p. 221) about physical activities that are relevant to their individual destiny in life. Students, especially those in secondary schools, are experienced learners and capable of interpreting and internalizing meanings in physical activities being taught in our classes. Thus, helping students internalize meaning and formulate meaningfulness may be a more important task for us than merely teaching the activity. In other words, we need to help students transform the purpose of an activity into a personal desire, turn their interest in an activity into a personal striving, and bridge their knowledge and skill into willingness so that they will continuously pursue a physically active life style.

\section{References}

Bye, L., \& Jussim, L. (1993). A proposed model for the acquisition of social knowledge and social competence. Psychology in the School, 30, 143-161.

Chen, A. (1996). Student interest in activities in a secondary physical education curriculum: An analysis of student subjectivity. Research Quarterly for Exercise and Sport, 67, 424-432.

Chen, A. (1998). Perception of boredom: Students' resistance to a secondary physical education curriculum. Research in Middle Level Education Quarterly, 21, 1-20.

Cornbleth, C. (1990). Curriculum in context. Bristol, PA: Falmer Press.

Cronbach, L.J. (1951). Coefficient alpha and the internal structure of tests. Psychometrika, 16, 297-334.

Csikszentmihalyi, M. (1990). Flow: The psychology of optimal experience. New York: Harper \& Row.

Doll, W.E., Jr. (1992). A post-modern perspective on curriculum. New York: Teachers College Press.

Ennis, C.D. (1992). Curriculum theory as practiced: Case studies of operationalized value orientations. Journal of Teaching in Physical Education, 11, 358-375.

Goetz, J.P., \& LeCompte, M.D. (1984). Ethnography and qualitative design in educational research. San Diego, CA: Academic Press. 
Griffin, P.S. (1984). Girls' participation patterns in a middle-school team sports unit. Journal of Teaching in Physical Education, 4, 30-38.

Griffin, P.S. (1985). Boys' participation styles in a middle-school physical education team sports unit. Journal of Teaching in Physical Education, 4, 100-110.

Jewett, A.E., \& Bain, L.L. (Eds.). (1987). The purpose process curriculum framework: A personal meaning model for physical education. Journal of Teaching in Physical Education Monograph, 6, 195-366.

Jewett, A.E., Bain, L.L., \& Ennis, C.D. (1995). The curriculum process in physical education (2nd ed.). Dubuque, IA: Brown.

Joreskog, K.G., \& Sorbom, D. (1993). LISREL ${ }^{\circledR}$ 8: Structural equation modeling with the SIMPLIS ${ }^{\mathrm{TM}}$ command language. Hillsdale, NJ: Erlbaum.

Kaiser, H.F. (1974). An index of factorial simplicity. Psychometrika, 39, 31-36.

Kenyon, G.S. (1968a). A conceptual model for characterizing physical activity. The Research Quarterly, 39, 96-105.

Kenyon, G.S. (1968b). Six scales for assessing attitude toward physical activity. The Research Quarterly, 39, 556-574.

Kirk, D. (1993). Curriculum work in physical education: Beyond the objectives approach? Journal of Teaching in Physical Education, 12, 244-265.

Langley, D.J. (1995). Examining the personal experience of student skill learning: A narrative perspective. Research Quarterly for Exercise and Sport, 66, 116-128.

Lee, A.M., Landin, D.K., \& Carter, J.A. (1992). Student thoughts during tennis instruction. Journal of Teaching in Physical Education, 11, 256-267.

Lee, A.M., \& Solmon, M. (1992). Cognitive conceptions of teaching and learning motor skills. Quest, 44, 57-71.

Lirgg, C.D. (1994). Environmental perceptions of students in same-sex and coeducational physical education classes. Journal of Educational Psychology, 86, 183-192.

MacDonald, J.B. (1988). Theory, practice, and the hermeneutic circle. In W.F. Pinar (Ed.), Contemporary curriculum discourses (pp. 101-113). Scottsdale, AZ: Gorsuch, Scarisbrick.

Pinar, W.F., Reynolds, W.M., Slattery, P., \& Taubman, P.M. (1995). Understanding curriculum: An introduction to the study of historical and contemporary curriculum discourses. New York: Peter Lang.

Slattery, P. (1995). Curriculum development in the postmodern era. New York: Garland.

Stevens, J. (1992). Applied multivariate statistics for the social sciences (2nd ed.). Hillsdale, $\mathrm{NJ}$ : Erlbaum.

Strauss, A.L. (1987). Qualitative analysis for social scientists. New York: Cambridge.

\section{Appendix}

\section{Remaining Items in the Meaingfulness Scale after Confirmatory Factor Analyses}

Item

Social bonding

6. I want to play the sports that my boy/girl friend likes.

19. I want to learn those sports popular among my friends.

29. I want to play those sports where I can be part of a team.

43. I want to contribute my talent to my team in physical education.

61. I want to learn the social skills of making friends when playing sports. 
Cultural appreciation

7. I want to learn those sports from different cultures.

25. I enjoy doing activities that have local cultural stories behind them.

31. I practice the way a sport is played by people in its original culture.

49. I want to play those sports that involve learning about other cultures.

\section{Challenge}

45. I always want to be challenged in physical education.

52. I want to learn those sports that involve playing complex skills.

53. I exercise in physical education to challenge my physical potential.

56. I enjoy learning competitive Olympic sports in physical education.

59. I can practice a drill repeatedly even when I keep failing at it.

Tension release

33. I play rigorously in physical education to relax my mind.

37. I believe that playing sports helps me relax in school.

38. I play sports in physical education to ease my mental tension.

Fitness development

24. I exercise in physical education to help my heart function well.

28. I do exercises in physical education to control my weight.

47. I want to do those sports that help build my muscles.

65. I want to play anything that can help pump my heart fast.

Self -expression

1. I practice repeatedly to improve the artistic expression of my movement.

16. I play hard in physical education to display my emotions.

17. I want to show my friends my talent in sport classes.

66. I enjoy playing those sports that help others appreciate my feelings. 